\title{
Research on the model of Human-Robot football professional skills competition
}

\author{
Yong Ding ${ }^{1, a}$ Jiefang Liu $^{2}$ \\ ${ }^{1}$ Hubei Communications Technical College, Public Class Department, No. 455 Wuhan Xiong Chu Street, China \\ ${ }^{2}$ Hubei Communications Technical College, Traffic Information Department, No. 455 Wuhan Xiong Chu Street, China
}

\begin{abstract}
In this paper, the robot (humanoid robot) soccer activity is decomposed according to the process of Human-Robot football professional skill competition. This paper analyzes the shooting problem of robot in the forward range and outside the range of the door frame, applies it to the rotation angle of robot foot, and gives the mathematical model of linear motion through the force of the center of the ball. Then, the further research direction are discussed.
\end{abstract}

\section{Problems of Human-Robot football professional skill competition}

The World Cup is an influential event in the world. It can show people the latest achievements and applications of science and technology in the World Cup. With the advent of the era of artificial intelligence, Intelligent computing and signal processing technology are displayed in front of people. The 2018 World Cup in Russia has added VAR (Video Assistant Referee)[1]. The official designation of the ball has embedded a smart chip into it, making football a smart football. At present, human football is mainly played on the field. Although robot football is mainly played, it is mainly battles between robots. Football matches between humans and robots are also played. Not in real life, and Google's recent push for the man-machine game of Go has increased the author's interest in the study of humancomputer football.

In this paper, the robot (humanoid robot) activities in the Human-Robot soccer match are decomposed according to the process of the game. The following aspects are studied:

(1) Appearance model research;

(2) Research on kick-off model;

(3) Research on passing and steals model;

(4) Shooting model research.

The research can be applied to daily club leagues, the Intercontinental Cup and the World Cup.

\section{Preparations for model research}

Assuming the height of the robot $i$ is $h_{\mathrm{i}}$ meters, the robot's eyes are equipped with sensors to sense the position of intelligent soccer. The plane of the football field is regarded as a plane rectangular coordinate system, and the white lower left corner (the boundary point of the corner ball) in Figure 1 below is the origin of the coordinate system. If the point is crossed as the vertical line of the court plane, the vertical coordinate axis of the space rectangular coordinate system can be obtained, thus setting the space coordinates of the points on the court (including the points on the goal) and the football ball ball. The spatial coordinates of any point on the ground or in the air.

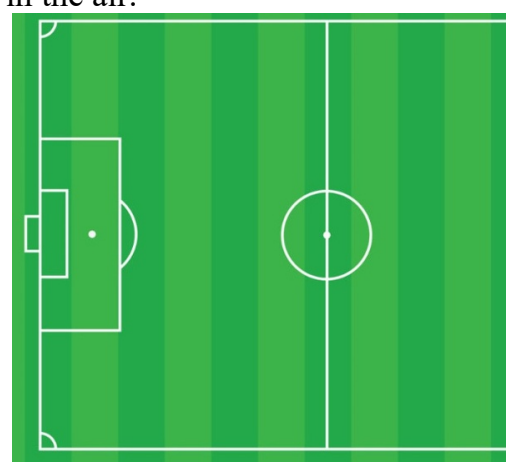

Figure 1. Football half court plan.

\section{Model Research}

\subsection{Research on appearance model}

Suppose that in order to save energy, humans place the robots in a vertical queue in an approach waiting area.

At the same time, start all the batteries inside the robot, so that the robot forward at a uniform speed, line up from the off-court channel into the court, and arrive at the center of the court queue (the first to enter the court is vertical queue, the first robot to reach the designated position to turn, the other robots in turn on its side of the queue crosswise. At the same time turn around, then all robots waved to the audience like humans. This procedure can be described as follows:

(1) The first robot goes to the designated position and stops and turns

\footnotetext{
a Corresponding author: ding_yong_maths@126.com
} 
In this process, the designated position refers to the turning position of most human team members. The robot can determine the designated position independently, that is, align with the line after the human pause, which can be realized by the robot's eye sensor. After the robot pause, the whole body should rotate 180 degrees clockwise, and rotate the whole body through clockwise rotation of the right foot.

(2) All robots waved to the auditorium spectators

After the first step, the robot is already facing the audience. At this point, the robot's internal mechanical device starts the arm-raising operation, and starts the expression change, smiling to the audience. With the help of face recognition function, the robot can identify the audience in the stands (i.e. determine where there are people in the stands and where there are no people) and wave in the direction of the audience. During the wave, the robot rotates its head (The rotation range can be set to angle $[-60,60]$ with the positive axis as the center, where the negative sign indicates counterclockwise rotation, otherwise clockwise rotation).

\subsection{Research on kick-off model}

In order to maintain the dominant position of human beings, all referees are set as human beings. Combining with the rules of football matches, the kick-off process can be described as follows:

(1) The referee toss a coin, and the robot guess the result

The robot waits for the referee to toss the coin, then decides that the referee has finished the toss, guesses the toss result randomly and gives out the voice. If the robot knows through the ear sensor that the referee said "correct", then the random reply "our first half attack direction is forward" or "our first half attack direction is forward rear"; if the robot knows through the ear sensor that the referee said "wrong", then waits for the human reply "our first half attack direction is forward" Forward "or" our attack direction in the first half is backward ", the robot judges its own attack direction according to the human answer (contrary to the attack direction of the human answer), and then the robot kicks off.

(2) Kick-off

If the robot does not guess in step (1), that is, the ball is kicked off by the robot, the robot will take the ball, and according to the eye sensor to determine the location of the center of the stadium marking, this method is to place a sensor in the center of the stadium marking position, or directly according to the center marking in the three-dimensional rectangular coordinate system. Position point coordinates to judge. Assuming that the football field is $2 a$ meters long and $2 b$ meters wide, the coordinates of the center marking of the field are $(a, b)$, and the robot tells the robot arm to place the ball on the center marking of the field through an eye sensor.

If the robot has guessed in step (1), that is, the human kick-off, then the robot player immediately starts the robot foot walking after the random answer in step (1).

Let the foot of the robot $i$ be $c_{\mathrm{i}}$ meters away from the straight line of the intelligent soccer ball. If $c_{\mathrm{i}}<c$ (usually $c=9.15$ ), the robot moves itself to the position from the ball $c$ meters and stops and waits for the kickoff. To imitate humans, assume that only sensors are installed in the eyes, and that the height of the robot $i$ 's eye sensor is $h_{\mathrm{i}}{ }^{*}$ meters, the eye sensor's linear distance from the smart football is:

$$
d_{i}^{*}=\sqrt{c_{i}^{2}+h_{i}^{* 2}}
$$

When the robot is satisfied $c_{\mathrm{i}} \geqq c$, the robot's eye sensor detects that it stops walking and waits for the human kick-off when the linear distance from the intelligent football reaches or exceeds: $\sqrt{c^{2}+h_{i}^{* 2}}$.

\subsection{Research on passing and steals model}

Robot ball passing can include: inner instep, arch, outer instep, toe, heel and other ways, these ways are carried out by the robot itself, robot behavior by the robot brain chip to control the movement of mechanical parts of the whole body.

Assuming a robot kick-off, the robot directs the foot pass directly to the nearest teammate (force directed toward the teammate and control the force), and then the robot determines the direction of the human goal (sensors are installed below both ends of the goal) to determine that there is no human player (obstacle) in the horizontal direction closer to the goal and between the two robots. Assuming that the ball is played by humans, the robot will begin to steal it. Assuming that the robot defaults to cooperative defense, the robot will try its best to steal the ball. That is, when the ball is at the foot of humans, the robot will steal the ball by two humans, the nearest two straight distances from the ball. Robots jointly steal.

Generally, the way of steals in football matches are: front steals, side steals and rear steals. Robots can take any kind of robbery, but to avoid physical contact with people, that is, to avoid harm to people. In order to avoid human injury, it is stipulated that a robot can not slide a shovel. If the robot has shoveled a human for some reasons and has shoveled a human down, it will be penalized for a foul. The robot receives the signal sent by the referee (human) for a foul (the referee is equipped with an instrument to click a button to penalize the foul). Stimulate a helpless expression on the face and spread out your hands to express regret (if you need to save money, you can not design an expression change function for the robot), and stop walking for a while until the referee presses the signal button to restart the game.

\subsection{Research on shooting model}

When the space around the robot $i$ is sufficient (e.g. the recognition result detected by the eye face recognition function within the cylindrical area with a set neighborhood radius $r_{\mathrm{i}}$ is "no human"), the ball can be headed or shot from a foot, otherwise passing.

The position, angle, force and speed of the robot head or foot determine the accuracy of the goal. Assuming that the robot can choose to pass or shoot the ball by itself, if the eye sensor coordinates of the robot $i$ are ( $x_{\mathrm{i}}$, 
$\left.y_{\mathrm{i}}, h_{\mathrm{i}}{ }^{*}\right)$, then the foot coordinates of the second robot are $\left(x_{i}, y_{i}, 0\right)$ : the robot should be able to use the foot to catch the ball smoothly. The position in which the ball can be touched is similar to that of a missile interceptor (the foot is similar to an interceptor missile and the ball is similar to an interceptor missile). The vertical coordinates of the foot rise, the horizontal coordinates and the vertical coordinates also change during the leg lifting. To achieve the precise change of the coordinates, the corresponding precise movement of the legs is required, and the line of the legs will be straight. Lines become polygonal lines, in which the rotation of the thigh and calf axes occurs, which can be analyzed by the rotation matrix.

Suppose the ball is stepped on the foot of the robot after receiving the ball (making it close to 0 ) and then swinging its legs towards the goal to shoot. The speed of the football leaving the robot's foot is $v_{\mathrm{i}}$ meters per second (the speed of the ball can be measured and recorded by the chip in the intelligent football, which is related to the amplitude and speed of the robot's swinging legs). The acceleration of the ball is $a_{\mathrm{i}}$ meters per square seconds, and the mass of the ball is $m$ kilogram. The data stored in the intelligent football include time data of robot foot contact at each time period ( Suppose the first contact time of the robot $i$ contact is $t_{\mathrm{ij}}$, and the end of contact is $t_{\mathrm{ik}}, j$ indicates that $n$th time touched the ball, and $k$ indicated the end of $n$th time touched the ball ). The force of the robot $i$ on the ball is

$$
F_{i}=m a_{i}=m \frac{\Delta v_{i}}{\Delta t_{i}}=\frac{m v_{i}}{t_{i k}-t_{i j}}
$$

If the force passes through the center of the ball, the ball moves forward in a straight line, and if the force does not pass through the center of the ball, the ball moves forward in an arc. The speed of the ball is reduced by the influence of air resistance and self weight.

The main force of the study is discussed in three aspects: the shape of the ball, the linear motion, and the following.

(1) The robot shoots in the direction opposite to the doorframe

According to the foregoing Figure 1, the intersection point of the white line at the lower left corner (the farthest corner corner) is the coordinate origin. If the force passes through the center of the sphere, the ball moves forward in a straight line. In order to ensure human safety, the robot should not shoot too fast after shooting, that is, the robot should control its own kicking force, but should not be too slow, otherwise it will be easily saved by human goalkeepers or blocked by human players. The robot needs to judge the inclined direction of the human goalkeeper's body beforehand, so it can finish the goal by shooting in the other direction. That is, the robot needs to recognize the human goalkeeper's posture. The robot pauses for a moment before shooting, waiting for the human goalkeeper to show a propensity to save for posture recognition before shooting.

Suppose the human goal is on the left side of the goal in Figure 1. In Figure 1, the direction from the origin to the right is the positive direction of the $\mathrm{X}$ axis, and the direction from the origin to the upward is the positive direction of the $\mathrm{Y}$ axis.

If the robot's eye sensor recognizes that the human goalkeeper's propensity to save is in the positive direction toward the $\mathrm{Y}$ axis, the robot shoots in different directions. At this point, the robot, whose field of vision is perpendicular to the goal, shoots the right foot clockwise rotating angle $\theta_{i}$ through the back of the inner foot, that is, the angle between the extension line of the right foot and the positive direction of the $\mathrm{X}$ axis is $\left(\pi-\theta_{i}\right)$. Assuming that the goal length is $2 a$ ' (usually 7.32 meters), the coordinates of the border point A near the origin is $(0, \mathrm{a}-\mathrm{a}, 0)$. The coordinates of the foot $\mathrm{B}$ of the robot $i$ are $\left(x_{\mathrm{i}}, y_{\mathrm{i}}, 0\right)$. The robot's eye sensor can sense the vertical distance $\left(x_{\mathrm{i}}\right)$ between the robot and the goal. The angle between the line segment $\mathrm{AB}$ and the goal line is $\theta_{i}^{*}$, then:

$$
\begin{gathered}
\sin \theta_{i}^{*}=\frac{x_{i}}{\|A B\|} \\
\Rightarrow \theta_{i}^{*}=\arcsin \frac{x_{i}}{\sqrt{x_{i}^{2}+\left(y_{i}-a+a^{\prime}\right)^{2}}}
\end{gathered}
$$

The robot's right foot rotates clockwise at least more than the angle above (otherwise it will kick the ball out of the frame) and will not shoot in the direction of the goalkeeper's save. Otherwise, it will kick the ball out of the frame, which can lead to a goal if

$$
\theta_{i}^{*}<\theta_{i}<\frac{\pi}{2}
$$

is met.

Similarly, if the robot's eye sensor recognizes that the human goalkeeper's propensity to save is toward the $y$ axis, the robot shoots in different directions. At this point, the robot whose field of vision is perpendicular to the goal shoots the right foot anti-clockwise rotation angle $\alpha_{i}$ through the back of the outer foot, and the coordinates $\left(0, \mathrm{a}^{+} \mathrm{a}^{\prime}, 0\right)$ of $\mathrm{A}^{\prime}$. The angle between the line segment A'B and the goal line is $\alpha_{i}^{*}$, then:

$$
\alpha_{i}^{*}=\arcsin \frac{x_{i}}{\sqrt{x_{i}^{2}+\left(y_{i}-a-a^{\prime}\right)^{2}}}
$$

The robot's right foot rotates counterclockwise at least beyond the angle above (otherwise it will kick the ball out of the frame) and does not shoot in the direction of the goalkeeper's save to get a goal, that is to say, a goal can be scored when

$$
\alpha_{i}^{*}<\alpha_{i}<\frac{\pi}{2}
$$

is met.

(2) The robot shoots at the position of the left side facing the doorframe opposite to the range

In this case, if the robot does not rotate the whole body, the robot can only shoot through the back of the outside foot, assuming that the robot's foot rotates counterclockwise at an angle of $\beta_{i}$. 
If the human goalkeeper's dive tendency is toward the $y$-axis and the human goalkeeper's initial position is in the middle of the goal, i.e. AA's midpoint A" $(0, a, 0)$. Then:

$$
\begin{gathered}
\left\{\begin{array}{l}
\sin \beta_{i}^{*}=\frac{x_{i}}{\|A B\|} \\
\sin \tilde{\beta}_{i}=\frac{x_{i}}{\left\|A^{\prime \prime} B\right\|}
\end{array}\right. \\
\Rightarrow\left\{\begin{array}{l}
\beta_{i}^{*}=\arcsin \frac{x_{i}}{\sqrt{x_{i}^{2}+\left(y_{i}-a+a^{\prime}\right)^{2}}} \\
\tilde{\beta}_{i}=\arcsin \frac{x_{i}}{\sqrt{x_{i}^{2}+\left(y_{i}-a\right)^{2}}}
\end{array}\right.
\end{gathered}
$$

The rotation angle should be between the above two to score (can not take the endpoint, because the endpoint corresponds to the post and goalkeeper), that is, meet $\tilde{\beta}_{i}<\beta_{i}<\beta_{i}^{*}$ can lead to the goal.

Similarly, if the goalkeepers' propensity to fight is toward the negative direction of the $\mathrm{Y}$ axis, then:

$$
\hat{\beta_{i}}=\arcsin \frac{x_{i}}{\sqrt{x_{i}^{2}+\left(y_{i}-a-a^{\prime}\right)^{2}}}
$$

Turning angle meets $\hat{\beta}_{i}<\beta_{i}<\tilde{\beta}_{i}$ can lead to goals.

(3) The robot shoots at the right side of the doorframe opposite the range

In this case, the robot can shoot through the instep or arch of the foot, assuming that the robot's foot rotates clockwise at an angle of $\gamma_{i}$.

Similarly, if the human goalkeeper's propensity to save is in the positive direction of the y-axis (the robot's eye sensor perceives that it is moving to the right), then:

$$
\begin{gathered}
\left\{\begin{array}{l}
\sin \gamma_{i}^{*}=\frac{x_{i}}{\left\|A^{\prime \prime} B\right\|} \quad(11) \\
\sin \gamma_{i}=\frac{x_{i}}{\|A B\|}
\end{array}\right. \\
\Rightarrow\left\{\begin{array}{l}
\gamma_{i}^{*}=\arcsin \frac{x_{i}}{\sqrt{x_{i}^{2}+\left(y_{i}-a\right)^{2}}} \\
\tilde{\gamma}_{i}=\arcsin \frac{x_{i}}{\sqrt{x_{i}^{2}+\left(y_{i}-a+a^{\prime}\right)^{2}}}
\end{array}\right.
\end{gathered}
$$

Turning angle meets $\tilde{\gamma}_{i}<\gamma_{i}<\gamma_{i}^{*}$ can lead to goals.

If the human goalkeeper's dive tends to be negative on the y-axis (the robot's eye sensor senses it is moving to the left), then there is

$$
\hat{\gamma_{i}}=\arcsin \frac{x_{i}}{\sqrt{x_{i}^{2}+\left(y_{i}-a-a^{\prime}\right)^{2}}}
$$

Turning angle meets $\gamma_{i}^{*}<\gamma_{i}<\hat{\gamma}_{i}$ can lead to goals.

\section{Conclusion and Further research}

Conclusion is as follows:

In the case of robot sensors installed in the eye, the model given in this paper can improve the goal rate.

Further research in the future will include:

(1) Set-pieces shooting appeared many times in the World Cup in 2018, mainly including corner kicks, free kicks, penalties, etc., which can be further studied.

(2) The subsequent links (celebration, rescue and departure) can be used for further research.

(3) Unification of clockwise and counter clockwise angles and the simulation and error analysis of the model.

(4) The study of the force acting in this article does not pass through the shape of the ball (arc motion).

(5) Gesture recognition research [2].

(6) Robot soccer team based on brain like computation.

(7) Research on wonderful goals [3].

Brain-like computing[4,5] is the difficulty and trend of artificial intelligence research. Researchers are looking forward to the development of artificial brain which is close to the function of human brain.

\section{Acknowledgements}

This research was financially supported by The General Project of Hubei Educational Science Planning "Research on the Reform of Vocational Skills Competition Promoting Talents Cultivation Model" (Project No. 2017GB189).

\section{References}

1. Sohu. The world cup you see is actually the battlefield of AI.

http://www.sohu.com/a/239282045 99988082 (2018)

2. Kai Tian. Research on the interactive technology of human and medium-sized soccer robot competition. http://www.wanfangdata.com.cn/details/detail.do? t ype $=$ degree \&id $=$ D01088435 (2018)

3. FIFA. Top 10 Goals: Russia 2018. https://www.fifa.com/worldcup/ (2018)

4. Qian Liang. Computational method based on brain like multi sensory attention switching. http://dbpub.cnki.net/grid2008/dbpub/detail.aspx?db $\underline{\text { code }}=$ SCPD \&dbname $=$ SCPD2018\& filename $=\mathrm{CN} 1$ 08197711A (2018)

5. 52jingsai. 2018 international undergraduate brain computing innovation application contest. http://www.52jingsai.com/article-5284-1.html (2018) 